\title{
Numerical aspects of nonlinear flexible aircraft flight dynamics modeling
}

\author{
Robert J. S. Simpson* and Rafael Palacios ${ }^{\dagger}$ \\ Imperial College, London, SW7 2AZ, United Kingdom
}

\begin{abstract}
A critical review of the numerical approximations made in flexible aircraft dynamics modeling is presented. The baseline model is a geometricallyexact composite beam model describing the flexible-body dynamics which are subject to aerodynamic forces predicted using the unsteady vortexlattice method (UVLM). The objectivity of the beam formulation is first investigated for static problems with large nodal rotations. It is found that errors associated with non-objectivity of the formulation are minimized to negligible levels using quadratic (3-noded) elements. In addition to this, two force calculation methods are presented and compared for the UVLM. They show subtle but important differences when applied to unsteady aerodynamic problems with large displacements. Nonlinear static aeroelastic analysis of a very flexible high-altitude long-endurance (HALE) wing is also carried out, and time-marching analysis is applied to the Goland wing in order to predict to the response at, and around, the flutter velocity. Conclusions drawn from the studies in this work work are directly applicable in the identification of appropriate modeling strategies in nonlinear flexible aircraft flight dynamics simulations.
\end{abstract}

\section{Nomenclature}

A panel area, $\mathrm{m}^{2}$

$\mathcal{A}$ aerodynamic influence coefficient matrix

$b \quad$ semi-chord, $\mathrm{m}$

$\Delta b \quad$ panel span, $\mathrm{m}$

\footnotetext{
*Graduate Student, Department of Aeronautics, 363A Roderic Hill Building. AIAA Student Member.

†Senior Lecturer, Department of Aeronautics, 355 Roderic Hill Building; rpalacio@imperial.ac.uk. AIAA Member.
} 


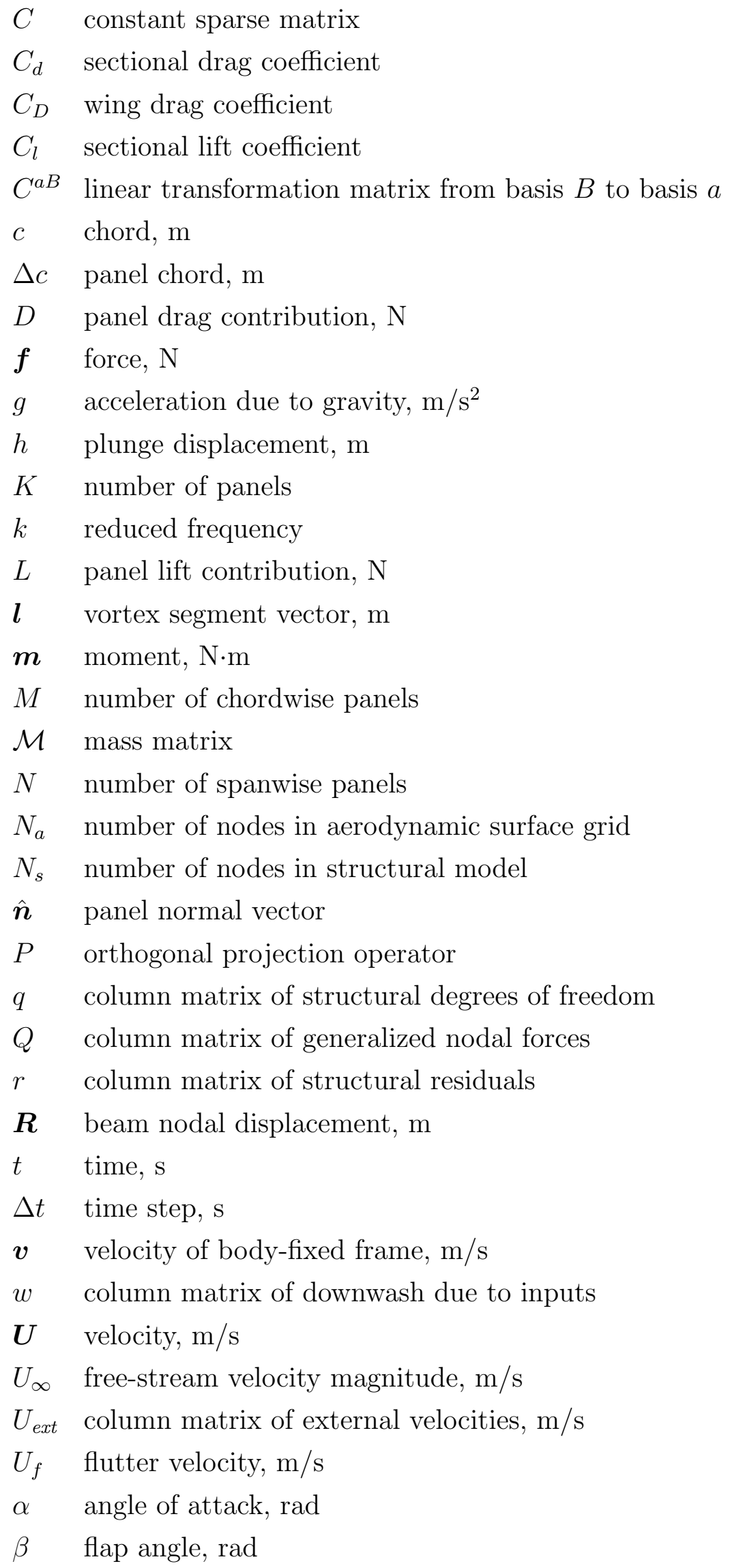




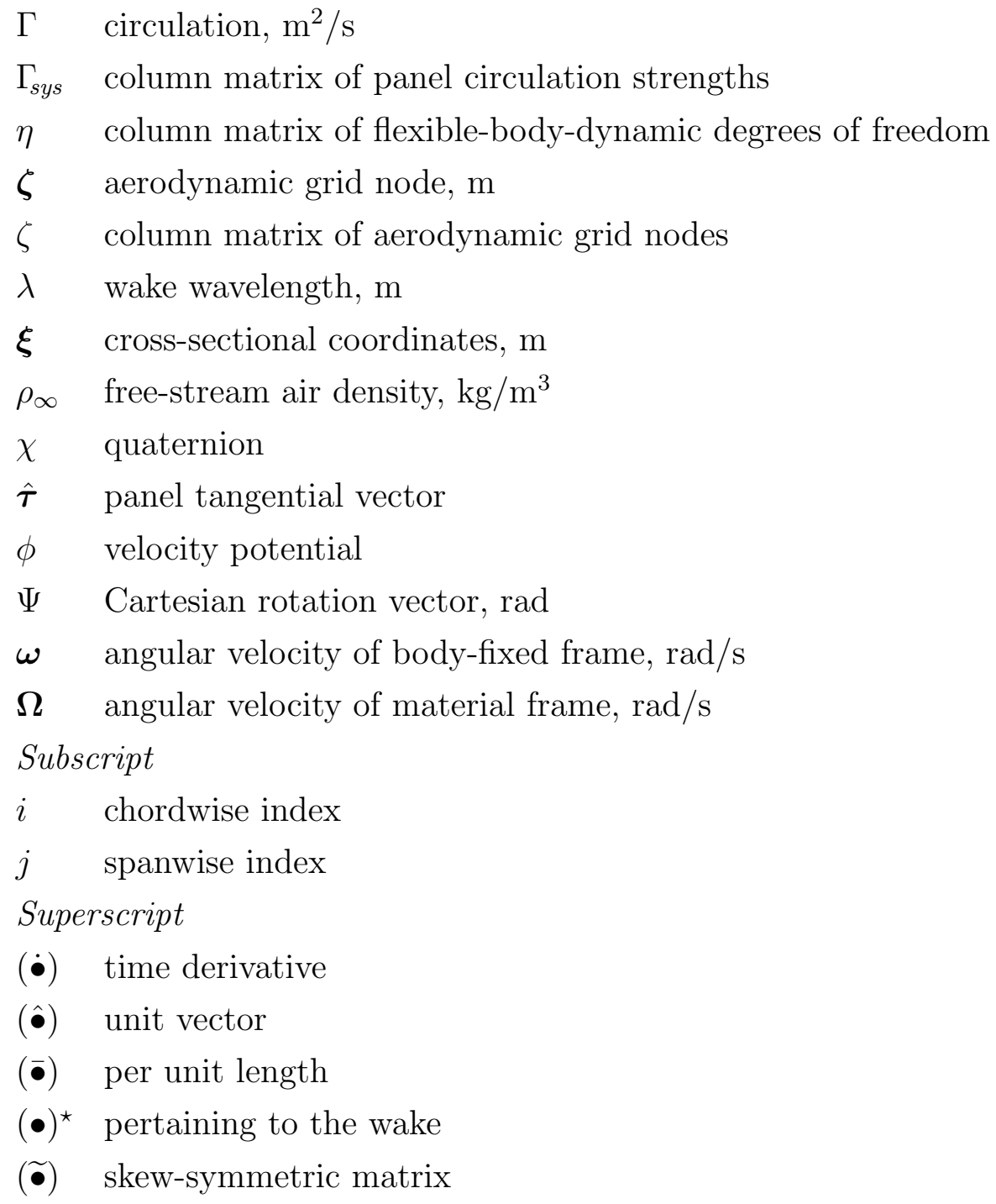

\section{Introduction}

This work seeks to address various numerical issues that arise in the nonlinear timedomain analysis of very flexible aircraft dynamics. Such analysis was deemed necessary following the Helios mishap report, ${ }^{1}$ which found that uncoupled linear treatment of a high-altitude long-endurance (HALE) aircraft's aeroservoelastic system was inadequate for predicting its dynamic behavior. Consequently, the coupled nonlinear analysis of flexible aircraft dynamics has been the focus of much research. Recent efforts have focused on coupling nonlinear beam formulations for structural dynamics with potential-based unsteady aerodynamics, ${ }^{2-6}$ and a similar approach is followed here.

HALE aircraft primary structures are generally slender and may exhibit nonlinear deformations, even in trim. ${ }^{7}$ Hence, nonlinear beam theories that include rigid-body degrees- 
of-freedom are often used in flexible aircraft flight dynamics (FAFD) analysis. ${ }^{2-6}$ A direct comparison between the available beam theories was presented by Palacios et al. ${ }^{8}$ In displacement-based formulations, difficulties associated with interpolating rotations can adversely affect the accuracy of solutions provided by beam models. ${ }^{9}$ These errors may be significant, particularly in a FAFD context, hence they are investigated here.

Low-to-medium fidelity aerodynamic models are favored for low-speed FAFD analysis due to their modest modeling effort and computational cost relative to higher-order, highfidelity models based on RANS equations. In particular, unsteady thin-airfoil theory ${ }^{10,11}$ is a two-dimensional linear theory in which the effect of shed vorticity is captured by a relative few state variables. This allows monolithic formulations of the aircraft aeroelastic problem; hence, two-dimensional finite-state aerodynamics have been used extensively in FAFD analysis. ${ }^{2,3,5,6}$ However, such approaches lack the ability to capture finite-wing effects, or the cross-influence of multiple surfaces and their wakes.

The unsteady vortex-lattice $\operatorname{method}^{12}$ (UVLM) is a three-dimensional, geometricallynonlinear method that can be used to calculate the unsteady aerodynamic loads on aircraft undergoing large dynamic deformations. In contrast to the doublet-lattice method ${ }^{13}$ (DLM) it is formulated in the time-domain, and the non-penetration boundary condition is enforced on the instantaneous deformed aircraft geometry. Finite-wing effects, large-deformations, and cross-influence of multiple lifting-surfaces can be captured with this method. The solution procedure leads to the creation of a shed wake, which may be allowed to evolve as a free-wake according to Helmholtz's laws of vortex motion. Hence, the UVLM is also suitable for, at least, the preliminary analysis of aerodynamic interference problems, ${ }^{14}$ and has been used to study wake-tail interference effects on the dynamics of HALE aircraft. ${ }^{15}$

There are some numerical aspects of the UVLM that are occasionally overlooked when the method is applied. Linear methods in aeroelasticity focus on lift forces and details of induced-drag calculation methods in the literature are rare and sometimes ambiguous. However, one needs to consider all the components of aerodynamic forces when large wing deformations are present and therefore good estimations of the induced drag are required. The details of suitable force calculation methods may have a significant effect on what constitutes acceptable mesh refinement.

The remainder of this paper will, firstly, outline the methods used for the flexible-body dynamics, aerodynamics, and the coupling of the two models. Detailed descriptions of these methods, including consistent linearization approaches, can be found in the work of Murua ${ }^{16}$ and Hesse. ${ }^{17}$ This paper is focused on the implementation of the nonlinear methods. Results are then presented for the independent structural and aerodynamic models before moving on to coupled aeroelastic analysis. The Goland wing ${ }^{18}$ and a representative HALE wing ${ }^{19}$ are analyzed in order to compare with existing results from the literature and to provide a basis 
for discussion. Particularly, it is the discretisation of the aerodynamic and structural models, and the details of the aerodynamic force calculation scheme, which will be discussed in the context of nonlinear static and dynamic aeroelastic analyses. Although no flight dynamics results are presented directly, results from the nonlinear aeroelastic model presented in this work can be readily appreciated from a FAFD modeling perspective, where the aeroelastic equations of motion are solved to predict aircraft behavior.

\section{Nonlinear Flexible-Body Dynamics: Displacement-Based Geometrically-Exact Composite Beam}

Primary structures of the aircraft are represented by curvilinear, composite (anisotropic) beams that may be initially curved and twisted, and are capable of large deformations and global rotations. Small local strains are assumed in the formulation. ${ }^{20}$ In this paper the following model will be referred to as the geometrically-exact composite beam (GECB) model. The beams are discretized using linear (2-noded) or quadratic (3-noded) finite elements. Each node has six primary degrees-of-freedom: The three components of nodal displacement, $\boldsymbol{R}_{a}$, where the subscript indicates that the components of vector $\boldsymbol{R}$ are expressed in the body-fixed frame, $a$; and the Cartesian rotation vector (CRV), $\Psi,{ }^{21}$ which describes the orientation of each node's local coordinate system, $B$, relative to the $a$-frame. For clarity, a column matrix of all the nodal displacements and rotations is defined, $\eta \in \mathbb{R}^{6\left(N_{s}-1\right)}$ where $N_{s}$ is the number of structural nodes. The flexible-body equations of motion are formulated in the $a$-frame, which is free to translate, $\boldsymbol{v}$, and rotate, $\boldsymbol{\omega}$, with respect to an inertial frame, $G$. Orientation of vector quantities in the local frame at each node, $B$, are projected into the $a$-frame by the linear transformation $C^{a B}(\Psi)$. An illustration of the frames of reference used in the formulation is shown in Fig. 1.

Using Hamilton's principle, the flexible-body equations of motion can be written in discrete form as, ${ }^{8}$

$$
\mathcal{M}(\eta)\left\{\begin{array}{c}
\ddot{\eta} \\
\dot{\boldsymbol{v}} \\
\dot{\boldsymbol{\omega}}
\end{array}\right\}+Q_{g y r}(\eta, \dot{\eta}, \boldsymbol{v}, \boldsymbol{\omega})+Q_{\text {stiff }}(\eta)=Q_{e x t}(\eta, \dot{\eta}, \boldsymbol{v}, \boldsymbol{\omega}, \chi)
$$

where matrix $M$ is the tangent mass matrix, and $Q_{g y r}, Q_{\text {stiff, }}$ and $Q_{\text {ext }}$ are the discrete gyroscopic, stiffness, and external generalized forces, respectively. Quaternions, $\chi$, are used to describe the orientation of the $a$-frame with respect to the inertial frame.

The discrete system is built upon finite-element interpolation within each element of the corresponding nodal degrees of freedom. In particular, interpolation of the Cartesian rotation 


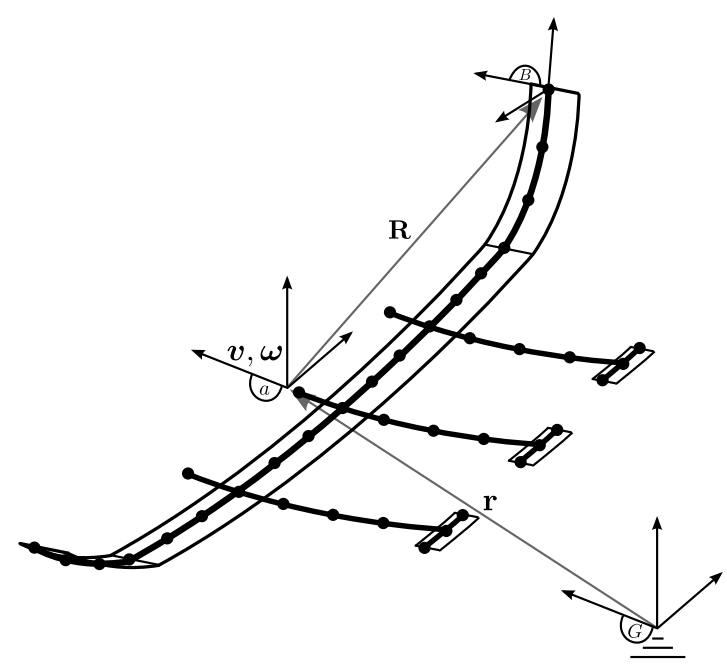

Figure 1. HALE aircraft represented by lifting-surfaces and beams. Frames of reference used in the flexible-body dynamics model are shown.

vector should guarantee invariance of the strain field under rigid-body rotations, ${ }^{9,22}$ otherwise known as objectivity. Alternative parameterizations of rotation are common, however the issue of objectivity remains regardless of which is used. Since rotation vectors are non-additive, the direct interpolation of rotation parameters leads to non-objectivity of interpolated strain measurements. ${ }^{9}$ Even the initial orientation of an undeformed beam can adversely effect the accuracy of predictions using displacement-based nonlinear beam theories. However, consideration of appropriate finite-element formulations and discretizations can minimize these errors. For example, using many frames of reference, one for each primary structure, reduces the proliferation of interpolation errors, ${ }^{17}$ however the transformations between frames may become an excessive burden on computational time. It is therefore desirable to use one frame for the definition of all the beam FEs, whilst attempting to quantify the level of error that may be introduced by the non-objectivity of the formulation.

\section{Aerodynamics: Unsteady Vortex-Lattice Method}

The unsteady vortex-lattice method (UVLM) calculates forces exerted on a streamlined body as it moves through an inviscid, incompressible fluid. Fluid particles in the majority of the domain are assumed to be irrotational, however rotational flow may exist within infinitesimally-thin shear layers on the body surface and in the trailing wake. Such flows may be modeled using potential flow, which is governed by Laplace's equation, $\nabla^{2} \phi=0$, where $\phi$, in this case, is a scalar-valued potential for the flow velocity, such that the flow velocity is given by $\boldsymbol{U}(\boldsymbol{x}, t)=\boldsymbol{\nabla} \phi(\boldsymbol{x}, t)$. The notation used to describe the UVLM in this work borrows heavily from that found in the thesis of Murua. ${ }^{23}$ 


\section{A. Solution Methodology}

A solution to Laplace's equation is sought by placing a lattice of rectilinear vortex rings over an infinitesimally-thin surface, which is used in place of the three-dimensional lifting surface geometry. Each vortex ring is a closed filament of vorticity with constant circulation strength, $\Gamma$, which induces a flow velocity calculated using the Biot-Savart law. ${ }^{12}$ Control points are chosen at the center of each vortex ring where the non-penetration boundary condition will be enforced; these are referred to as collocation points. A schematic of a simple lifting-surface is shown in Fig. 2, which illustrates the vortex lattice geometry and collocation point locations.

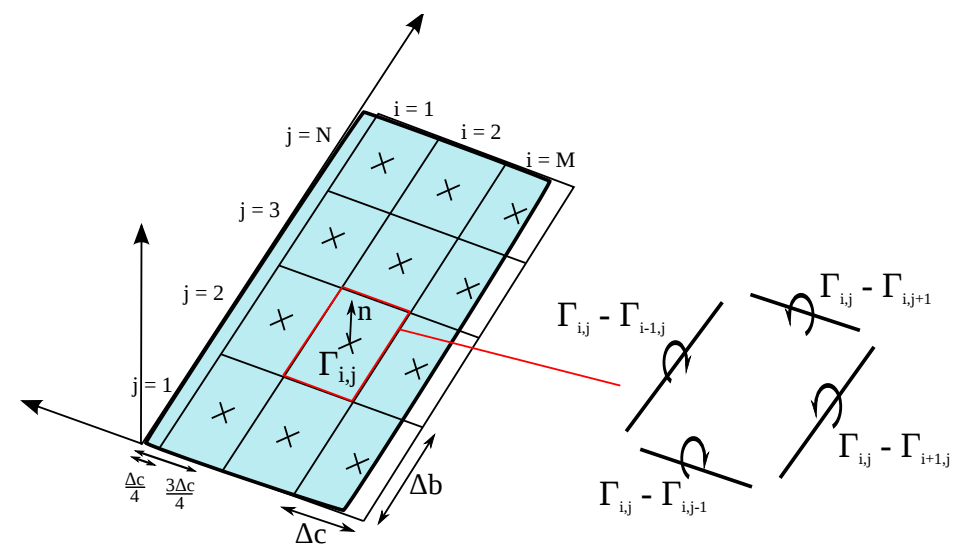

Figure 2. Body surface overlaid with vortex lattice geometry. Four vortex segments are shown on the right, which have circulation strengths equal to the difference in adjacent vortex ring strengths.

Enforcing the non-penetration condition, $\boldsymbol{U} \cdot \hat{\boldsymbol{n}}=0$, at each of the collocation points leads to a system of algebraic equations of the form,

$$
\mathcal{A} \Gamma_{\text {sys }}^{n+1}+\mathcal{A}^{\star} \Gamma_{s y s}^{\star^{n+1}}+w^{n+1}=\mathbf{0}
$$

where $\mathcal{A}\left(\zeta^{n+1}\right) \in \mathbb{R}^{K \times K}$ is the Aerodynamic Influence Coefficient (AIC) matrix, and $K=$ $M N$, where $M$ and $N$ are the number of chordwise and spanwise panels, respectively. Each element of the AIC matrix represents the normal velocity at one panel induced by another panel with all panel strengths set to unity; hence, it is purely function of the aerodynamic surface grid points, denoted $\zeta \in \mathbb{R}^{3 N_{a}}$ where $N_{a}$ is the number of surface grid points, and must be re-calculated should the aircraft deform. The superscripts $(\bullet)^{n}$ and $(\bullet)^{n+1}$ denote the time step. $\mathcal{A}^{\star}\left(\zeta^{n+1}, \zeta^{\star^{n+1}}\right) \in \mathbb{R}^{K \times K^{\star}}$ represents the normalwash induced by each wake vortex ring at the surface collocation points; it is analogous to $\mathcal{A}$, but is defined with one dimension of size $K^{\star}=M^{\star} N$, which is the number of wake panels, and $\zeta^{\star} \in \mathbb{R}^{3 N_{a}^{\star}}$, which are the wake grid points. $N_{a}^{\star}$ is the number of aerodynamic grid points in the wake. $\Gamma_{\text {sys }} \in \mathbb{R}^{K}$ and $\Gamma_{\text {sys }}^{\star} \in \mathbb{R}^{K^{\star}}$ are the column matrices of surface and wake circulation strengths, respectively. 
The downwash at the collocation points due to inputs, $w \in \mathbb{R}^{K}$, is given by $w=W\left[\dot{\zeta}+U_{\text {ext }}\right]$, where $U_{\text {ext }}$ are prescribed fluid velocities at the surface grid points which may include freestream velocities and gusts. The matrix $W\left(\zeta^{n+1}\right)$ interpolates from grid corner-points to collocation points and projects the resulting velocities along the corresponding panel normal vector.

As mentioned earlier the position and strength of a free wake is calculated by the UVLM. The propagation of circulation strengths through the wake is given by,

$$
\Gamma_{s y s}^{\star^{n+1}}=C_{\Gamma} \Gamma_{s y s}^{n}+C_{\Gamma}^{\star} \Gamma_{s y s}^{\star^{n}}
$$

where $C_{\Gamma} \in \mathbb{Z}^{K^{\star} \times K}$ is a sparse constant matrix that propagates the circulation strength from the trailing-edge at the previous time step into the first row of wake vortex rings. Analogously, $C_{\Gamma}^{\star} \in \mathbb{Z}^{K^{\star} \times K^{\star}}$ shifts the circulation strength in the wake downstream. The propagation of the wake grid, denoted $\zeta^{\star} \in \mathbb{R}^{3 N_{a}^{\star}}$ where $N_{a}^{\star}$ is the number of wake grid points, is given by,

$$
\zeta^{\star^{n+1}}-C_{\zeta} \zeta^{n+1}=C_{\zeta}^{\star} \zeta^{\star^{n}}+\Delta t\left(\mathcal{A}_{U} \Gamma_{s y s}^{n}+\mathcal{A}_{U}^{\star} \Gamma_{s y s}^{\star^{n}}\right)+\Delta t U_{\text {ext }}^{\star^{n}},
$$

where $C_{\zeta} \in \mathbb{Z}^{3 N_{a}^{\star} \times 3 N_{a}}$ closes the wake grid with the trailing-edge points at the current time step. $C_{\zeta}^{\star} \in \mathbb{Z}^{3 N_{a}^{\star} \times 3 N_{a}^{\star}}$ shifts the wake grid points downstream, and $U_{e x t}^{\star^{n}} \in \mathbb{R}^{3 N_{a}^{\star}}$ are prescribed fluid velocities at the wake grid points. The matrices $\mathcal{A}_{\boldsymbol{U}} \in \mathbb{R}^{3 N_{a}^{\star} \times K}$ and $\mathcal{A}_{\boldsymbol{U}}^{\star} \in \mathbb{R}^{3 N_{a}^{\star} \times K^{\star}}$ account for the fluid velocities induced by the surface and wake vortex rings, respectively - including these velocities allows the UVLM to capture wake roll-up. The Joukowksi hypothesis, which requires that flow leaves the trailing-edge smoothly, i.e. trailing-edge vortex segments have zero circulation, is approximately satisfied by setting the newly-shed panel strengths equal to the corresponding trailing-edge panel strength at the previous time step, Eqns.(3) \& (4). This also approximately satisfies Kutta condition, which is the requirement for no pressure jump to exist at the trailing-edge. ${ }^{24}$ The particular form of Eqn. (4) corresponds to a first-order, explicit, time-stepping scheme, which is widely used for the UVLM. ${ }^{4,12,23}$ Classical vortex methods are unconditionally stable (Cottet et al., ${ }^{25}$ pg. 43 44) however in the UVLM, in which new wake panels are shed from the trailing-edge, there are some limitations on the time step. Since a newly-shed wake panel has a circulation equal to the corresponding trailing-edge panel at the previous time step it should also be of equal area. Often it is useful to define a non-dimensional time step, $\frac{\Delta t U_{\infty}}{c}$, to help achieve this. Then, assuming that the chord is split into $M$ equally-sized panels, the time step can be set as $\frac{\Delta t U_{\infty}}{c}=\frac{1}{M}$. 


\section{B. Evaluation of Forces}

The literature surrounding the UVLM lacks a thorough discussion of force calculation methods, particularly with regards to unsteady induced-drag calculations. Accurate prediction of unsteady induced-drag is of great importance in flexible aircraft flight dynamics and flapping flight applications ${ }^{26,27}$ in which all components of the aerodynamic forces play an important role.

The main difficulty associated with induced-drag calculations in thin lifting-surface methods is the inclusion of leading-edge suction effects. Capturing leading-edge suction effects in the UVLM requires that force contributions from all bound vortex segments (singularities) are included. Such contributions are quasi-steady in nature, and have the form,

$$
\delta \boldsymbol{f}_{s t}=\rho_{\infty} \Gamma(\boldsymbol{U} \times \delta \boldsymbol{l})
$$

where $\delta \boldsymbol{l}$ is an incremental length of vortex segment. The force calculated for linear vortex segments using Eqn. (5) is approximated to act at the midpoint of the segment. Since the UVLM resolves the mean aerodynamic surface the unsteady force component from Bernoulli's equation, orientated along the normal of each vortex ring (panel), can be found. ${ }^{28}$ This leads to an unsteady contribution from each panel,

$$
\boldsymbol{f}_{u_{i j}}=\rho_{\infty} \frac{\partial \Gamma_{i j}}{\partial t} A_{i j} \hat{\boldsymbol{n}}_{i j}
$$

where $A_{i j}$ is the panel area, $\hat{\boldsymbol{n}}_{i j}$ is the panel normal vector, and $i$ and $j$ are panel indices in the chordwise and spanwise directions respectively. The unsteady force, Eq. (6), is approximated to act at the midpoint of the panel's leading vortex segment. For the proceeding discussion, this method, in which the steady contribution from each vortex segment (5) and the unsteady component from each panel (6) are summed, will be referred to as the Joukowski method.

An approximation to the method above is given by Katz \& Plotkin, ${ }^{12}$ in which the unsteady Bernoulli equation is applied to the top and bottom surfaces of each panel to find the pressure jump, which results in a local lift contribution,

$$
\begin{aligned}
L_{i j}^{l o c a l}=\rho_{\infty} & \left(\left(\boldsymbol{U}_{i j}^{u}+\boldsymbol{U}_{i j}^{w}\right) \cdot \hat{\boldsymbol{\tau}}_{i j}^{c} \frac{\Gamma_{i j}-\Gamma_{i-1, j}}{\Delta c_{i j}}\right. \\
& \left.+\left(\boldsymbol{U}_{i j}^{u}+\boldsymbol{U}_{i j}^{w}\right) \cdot \hat{\boldsymbol{\tau}}_{i j}^{s} \frac{\Gamma_{i j}-\Gamma_{i, j-1}}{\Delta b_{i j}}+\frac{\partial \Gamma_{i j}}{\partial t}\right) A_{i j} \cos \alpha_{i j}
\end{aligned}
$$

where $\boldsymbol{U}_{i j}^{u}$ is the velocity at the collocation points due to aerodynamic system inputs, i.e $\boldsymbol{U}_{i j}^{u}=\boldsymbol{U}_{i j}^{e x t}-\dot{\boldsymbol{\zeta}}_{i j}^{c o l}$, where $\boldsymbol{U}_{i j}^{e x t}$ and $\dot{\boldsymbol{\zeta}}_{i j}^{c o l}$ are external fluid velocities (such as free-stream or gust velocities) and the motion of the aerodynamic surface, respectively. $\boldsymbol{U}_{i j}^{w}$ are the ve- 
locity contributions from the wake vorticity, at the collocation points. The contribution of bound vorticity is approximated by two components arising from the gradient of circulation along the direction of panel tangential vectors $\hat{\tau}_{i j}^{c}$ and $\hat{\tau}_{i j}^{s}$, in the chordwise and spanwise directions, respectively. Katz \& Plotkin ${ }^{12}$ note that the force resulting from the pressure jump does not include leading-edge suction effects and is only adequate for lift prediction. Hence, the local angle of attack $\alpha_{i j}$ is introduced, which for general motions is $\alpha_{i j}=\tan ^{-1}\left(\boldsymbol{U}_{i j}^{u} \cdot \hat{\boldsymbol{n}}_{i j} / \boldsymbol{U}_{i j}^{u} \cdot \hat{\boldsymbol{\tau}}_{i j}^{c}\right){ }^{26}$ This effectively generalizes the lift prediction presented by Katz \& Plotkin ${ }^{12}$ for complex kinematics.

The induced drag is then calculated using the component of downwash that acts along the local lift vector. The local lift vector is found by projecting the panel normal vector onto the plane perpendicular to the relative inertial velocity. This is achieved using an orthogonal projection operator, ${ }^{21} P_{\hat{\boldsymbol{U}}_{i j}^{u}}=I-\hat{\boldsymbol{U}}_{i j}^{u} \hat{\boldsymbol{U}}_{i j}^{u \top}$, where $I$ is the identity matrix. The local drag is then

$$
D_{i j}^{l o c a l}=\rho_{\infty}\left[-\left(\boldsymbol{U}_{i j}^{b c}+\boldsymbol{U}_{i j}^{w}\right) \cdot\left(P_{\hat{\boldsymbol{U}}_{i j}} \hat{\boldsymbol{n}}_{i j}\right)\left(\Gamma_{i j}-\Gamma_{i-1, j}\right) \Delta b_{i j}+\frac{\partial \Gamma_{i j}}{\partial t} A_{i j} \sin \alpha_{i j}\right]
$$

where the superscript $b c$ indicates a velocity is calculated considering bound, chordwiseorientated vorticity only. At the leading-edge $\Gamma_{i-1, j}$ is set to zero. The total force contribution from each panel is then, $\boldsymbol{f}_{i j}=D_{i j}^{\text {local }} \hat{\boldsymbol{U}}_{i j}^{u}+L_{i j}^{\text {local }} P_{\hat{\boldsymbol{U}}_{i j}{ }_{i j}} \hat{\boldsymbol{n}}_{i j}$. Note that Eqns. (7) \& (8) do not account for sideslip, which is seen to have an effect in the context of nonlinear aeroelasticity, below.

In this method, which will be referred to as the Katz and Plotkin method, the panel forces are calculated based on velocities calculated at the collocation points - not directly on the vortex segments - introducing additional discretization error. Also, only spanwiseorientated vorticity can contribute to the local drag term. This may have implications for the level of mesh refinement used in the aerodynamic model, in particular on control surfaces which typically feature very coarse paneling. Further comments on the suitability of both methods for accurately predicting unsteady induced-drag can be found in previous work by the authors. ${ }^{29}$

\section{Multi-Disciplinary Considerations}

In this section the coupling of structural and aerodynamic models is presented in three sections. Firstly, the definition of the discretized fluid-structure-interface is presented. This includes the mapping of beam nodal displacements and rotations to aerodynamic grid nodes, and subsequently the mapping of forces on the aerodynamic grid nodes back onto the beam nodes. Secondly, three methods of coupled time-integration are presented. Finally, the 
software architecture used to implement the models is presented, namely, Simulation of High-Aspect-Ratio Planes in Python (SHARPy).

\section{A. The Fluid-Structure Interface}

The coupling of structural and aerodynamic finite-element discretizations is achieved using the assumptions of rigid cross-sections and coincident discretizations in the spanwise direction. Therefore, each structural node has a corresponding cross section defined by the points,

$$
\boldsymbol{\xi}_{B_{i}}=\left[\begin{array}{lll}
0 & \xi_{y_{i}} & \xi_{z_{i}}
\end{array}\right]^{T} \text { for }\{i \in \mathbb{Z} \mid 1 \leq i \leq M+1\},
$$

where $M$, as mentioned in Sec. III.A, is the number of chordwise panels in the aerodynamic model. The aerodynamic grid is defined in the inertial G-frame, where each aerodynamic grid point is found as,

$$
\boldsymbol{\zeta}_{G_{i j}}=\boldsymbol{r}_{G}+C^{G a}(\chi)\left[\boldsymbol{R}_{a_{j}}+C^{a B}\left(\Psi_{j}\right) \boldsymbol{\xi}_{B_{i}}\right],
$$

where $\boldsymbol{r}_{G}$ is the origin of the $a$-frame, $C^{G a}$ is the linear transformation from the $a$-frame to the inertial frame deduced from the quaternion $\chi, \boldsymbol{R}_{a_{j}}$ is the deformed position of the $j$-th structural node, and $C^{a B}$ is the linear transformation from the material $B$-frame to the $a$-frame based on the CRV at the $j$-th structural node, $\Psi_{j}$. Similarly, the velocities of the aerodynamic grid points are,

$$
\dot{\boldsymbol{\zeta}}_{G_{i j}}=C^{G a}(\chi)\left[\boldsymbol{v}_{a}+\widetilde{\omega}_{a} \boldsymbol{R}_{a_{j}}+\dot{\boldsymbol{R}}_{a_{j}}+C^{a B}\left(\Psi_{j}\right) \widetilde{\Omega}_{B_{j}} \boldsymbol{\xi}_{B_{i}}\right],
$$

where $\boldsymbol{v}_{a}$ is the translational velocity of the $a$-frame. The matrix-valued skew-symmetric operator $(\widetilde{\bullet})$ is applied to $\boldsymbol{\omega}_{a}$ and $\boldsymbol{\Omega}_{B_{j}}$, which are the angular velocities of the $a$-frame and $j$-th structural node, respectively.

After calculating the forces on the nodes of the deformed aerodynamic grid, $\boldsymbol{f}_{i j}^{a}$, they are mapped back onto the beam nodes resulting in nodal forces, $\boldsymbol{f}_{j}^{s}$, and moments $\boldsymbol{m}_{j}^{s}$. Since there is one cross-section for each beam node, the nodal forces and moments are,

$$
\boldsymbol{f}_{j}^{s}=\sum_{i=1}^{M+1} \boldsymbol{f}_{i j}^{a},
$$

which is the sum of forces acting on the aerodynamic nodes belonging to the $j$-th crosssection, and,

$$
\boldsymbol{m}_{j}^{s}=\sum_{i=1}^{M+1} \widetilde{\xi}_{i j} \boldsymbol{f}_{i j}^{a}
$$


which the sum of moments caused by the forces acting at aerodynamic nodes in the $j$-th cross-section. Note that the vectors in Eqns. (12) \& (13) have not been projected into any particular frame of reference; this is because, depending on how the beam finite-element model is defined, the nodal forces and moments may be desired in the $B$-frame (material), $a$-frame (body-fixed), or $G$-frame (inertial). Care must be taken to transform the quantities in these equations appropriately - cross-sectional coordinates, $\boldsymbol{\xi}_{i j}$, are defined in the $B$-frame and aerodynamic forces, $\boldsymbol{f}_{i j}^{a}$, are calculated in the $G$-frame.

\section{B. Time-Integration}

Integration of the coupled system, Eqns. (1 - 4), in time is done using a partitioned procedure $^{30}$ whereby separate integration schemes are used to advance the structural and aerodynamic states. The structural equations are solved using the Newmark- $\beta$ scheme, which for each time step, $n$, uses Newton-Raphson sub-iterations, $k$, to achieve a converged solution to Eqn. (1). In a loosely-coupled scheme the structural system residual, $r$, has the form,

$$
r_{n+1}^{k}=M \ddot{q}_{n+1}^{k}+Q_{g y r_{n+1}}^{k}+Q_{s t i f f_{n+1}}^{k}-Q_{e x t_{n}}
$$

where $\ddot{q}=\left\{\begin{array}{lll}\ddot{\eta} & \dot{\boldsymbol{v}} & \dot{\boldsymbol{\omega}}\end{array}\right\}^{\top}$ are the second derivatives of the structural degrees of freedom. Eqn. (14) corresponds to a loosely-coupled time-integration scheme because the external forces are calculated once at the start of each time step, and are based on the system states at time step $n$. Therefore, Eqn. (1) is not strictly satisfied at time $n+1$ and the solution of structural and aerodynamic states are staggered.

In order to truly satisfy Eqn. (1) at any given time step, a tightly-coupled procedure must be adopted. A tightly-coupled procedure is one that ensures up-to-date (i.e at time $n+1)$ structural and aerodynamic states are used to satisfy Eqn. (1). One way to achieve this is to embed the updating of aerodynamic states, and hence external loads $Q_{\text {ext }}$, within the Newton-Raphson loop, leading to a structural residual of the form,

$$
r_{n+1}^{k}=M \ddot{q}_{n+1}^{k}+Q_{g y r_{n+1}}^{k}+Q_{s_{t i f f}}^{k}-Q_{e_{n+1}}^{k} .
$$

The convergence criteria is defined as $\epsilon_{1}=\left(\max r_{n+1}^{k}\right) /\left|\max r_{n+1}^{0}\right|$ whether a loosely-coupled, Eqn. (14), or tightly-coupled, Eqn.(15), procedure is used. Unless otherwise stated, $\epsilon_{1}=$ $10^{-5}$ is used for all calculations in this paper.

An alternative tightly-coupled procedure can be constructed by using an outer fluidstructure sub-iteration loop within each time-step ${ }^{31}$ in the manner shown in Fig. 3. The aeroelastic loop continues until the change in external forces is brought below a certain tolerance. This second convergence criteria is therefore defined as $\epsilon_{2}=\left(\max \Delta Q_{e^{\prime} t_{n+1}}^{k^{\prime}}\right) /\left|\max Q_{e^{2} t_{n+1}}^{0}\right|$ 
where the superscript corresponding to $k^{\prime}$ refers to the index used in the aeroelastic loop, and $\Delta$ denotes the difference between successive iterations.

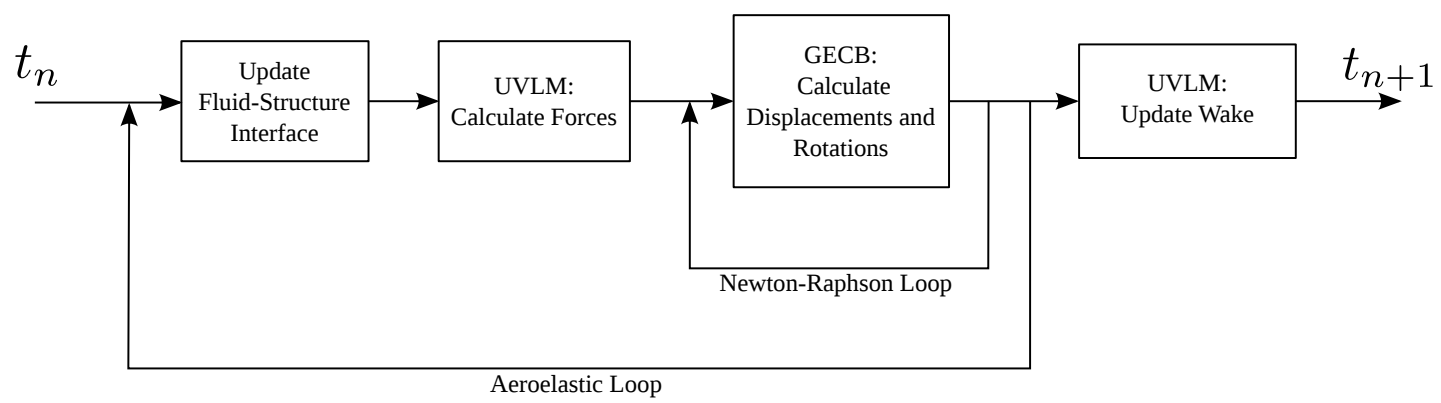

Figure 3. Outer fluid-structure coupling loop.

\section{Software Architecture}

The GECB, UVLM and fluid-structure interface models presented in this paper are implemented as stand-alone modules in the SHARPy framework. The framework is designed to be an efficient multi-disciplinary analysis tool, and as such leverages the advantages of several programming languages. In particular, the Python programming language is used to provide an interface between other Python-only modules, and shared libraries written in $\mathrm{C} / \mathrm{C}++$ and Fortran90. This approach is popular in the multi-disciplinary analysis and optimization community ${ }^{32-34}$ because it balances the ease of high-level development (in Python) with the efficiency of compiled code $(\mathrm{C} / \mathrm{C}++$ and Fortran) for computationally-expensive tasks. Care must be taken when sharing large amounts of data between modules because the cost associated with making local copies is prohibitive. In SHARPy, data is initialized in Python and pointers to this data shared between $\mathrm{C} / \mathrm{C}++$ and Fortran libraries using Python's built-in ctypes module. The UVLM can become computationally expensive when using a very high spatial or temporal resolution because the computational expense scales with the number aerodynamic degrees of freedom squared. Speeding up the UVLM with a fast panel method, ${ }^{35}$ a fast multipole method (FMM), ${ }^{36}$ or a combination of similar methods $^{37}$ has been carried out in the past by other authors. In this implementation it was found that parallelization using openMP was adequate to reduce simulation run-time to reasonable levels. 


\section{Numerical Studies}

In this section the objectivity of the beam formulation and the force calculation methods in the UVLM are investigated numerically and discussed in the context of FAFD modeling. These investigations inform an appropriate modeling approach for FAFD analysis using the methodologies presented in this paper. In particular, the objectivity investigation informs the choice of beam finite element, and the force calculation method informs what level of aerodynamic mesh refinement (paneling) is required. Finally, the analysis methodologies are exercised in the static and dynamic nonlinear aeroelasticity of very flexible wings.

\section{A. Objectivity Considerations in the Structural Model}

The test case used for the work presented here is defined in the book by Geradin and Cardona. ${ }^{21}$ It is a cantilever beam of length $L=5 \mathrm{~m}$, stiffness constants: $E A=4.8 \times 10^{8} \mathrm{~N}$, $G A=3.231 \times 10^{8} \mathrm{~N}$ (in both axes), $G J=1.0 \times 10^{6} \mathrm{Nm}, E I=9.346 \times 10^{6} \mathrm{Nm}$ (in both axes), and mass constants $\bar{m}=100 \mathrm{~kg} / \mathrm{m}$ and $J=10 \mathrm{kgm}$. Table 1 shows the geometricallynonlinear tip displacements for different discretizations under a dead vertical tip force of -600 $\mathrm{kN}$. The convergence parameter in the nonlinear static equilibrium is $10^{-5}$, and is defined as $\epsilon_{2}$ in Sec. IV.B where the superscript refers to a Newton-Raphson iteration on Eqn. (1) with velocities and accelerations set to zero. Convergence of computed tip displacements and rotations with published results is observed for both 2- and 3-noded elements as the number of elements increases.

\begin{tabular}{lrrr}
\hline Model & $\Delta R_{1}(\mathrm{~m})$ & $\Delta R_{3}(\mathrm{~m})$ & $\Psi_{2}(\mathrm{rad})$ \\
\hline 5 elements, 2 nodes & 0.586 & -2.147 & -0.6745 \\
10 elements, 2 nodes & 0.594 & -2.156 & -0.6726 \\
20 elements, 2 nodes & 0.596 & -2.159 & -0.6722 \\
2 elements, 3 nodes & 0.550 & -2.070 & -0.6576 \\
5 elements, 3 nodes & 0.589 & -2.144 & -0.6700 \\
10 elements, 3 nodes & 0.596 & -2.159 & -0.6719 \\
(Geradin and Cardona,2001) & & -2.159 & -0.6720 \\
\hline
\end{tabular}

Table 1. Tip displacements and rotations under tip dead force $(600 \mathrm{kN})$

Khosravi et al. ${ }^{38}$ provided a similar nonlinear test case for isotropic cantilevered structures subject to a tip moment, for which there is an analytical solution. The computed beam deformations should form an arc of constant radius which eventually becomes a circle under the application of increasing tip moments. The tip rotation resulting from a given tip moment, $M_{2}$, can be obtained analytically as $\Psi_{2}=\frac{L M_{2}}{E I}$. The analytical solution is matched 
almost perfectly by computed results, presented in Fig. 4. All results were obtained with 10 load sub-steps and a convergence parameter of $10^{-4}$.

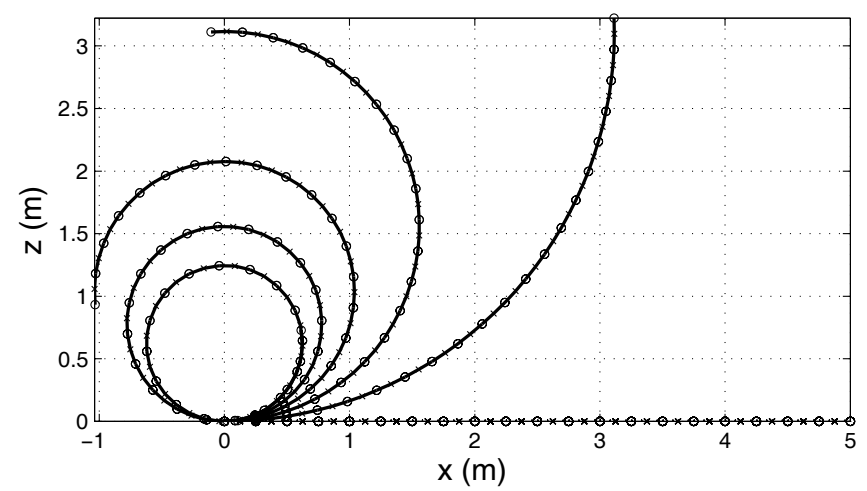

Figure 4. Application of a tip moment up to a maximum of $-15000 \mathrm{kNm}$, in increments of -3000 $k N .20$ 3-noded elements are used in all cases.

In both of the test cases presented above the beam deformation occurs in-plane, but specifically in a plane defined by the spatial (as opposed to material) coordinate system (in this case the $x-z$ plane). Consequently, the Cartesian rotation vector (CRV) has only one component and interpolation of rotations is not problematic. On inspection of Table 1 it appears that using either element-type leads to rapid convergence for such problems, and that the linear (2-noded) element may be slightly better. However, it will be seen that for practical problems, in which deformations are outside of any one plane defined by the spatial axes, the choice of element has a significant effect on the accuracy of interpolated rotations.

As mentioned above, the interpolation of rotations should guarantee objectivity, that is, invariance of the strain field under rigid-body rotations. ${ }^{9,22}$ This effect is investigated in the results of Table 2, which are obtained solving the cantilever beam under a tip following force of $3000 \mathrm{kN}$ for different azimuth locations, $\phi$, in the $x-y$ plane of the undeformed beam. The CRV in the solution includes the azimuth angle, and results in the table are rotated back to the plane of the undeformed beam. Both 2- and 3-noded elements are used in the analysis, and it should be noted that a model with $50 \times 3$-noded elements has the same number of degrees of freedom as one with $100 \times 2$-noded elements. As it can be seen, the quadratic interpolation on the three-noded element provides a much better approximation to the CRV than the linear interpolation on the two-noded element. The 3-noded quadratic elements are therefore favorable for our analysis.

\section{B. Force Calculation Methods in the Aerodynamic Model}

The unsteady lift and drag of a plunging airfoil is calculated using the UVLM and the results are shown in Fig. 5, where $h$ is the plunging amplitude and $b$ is the semi-chord. 


\begin{tabular}{llccc}
\hline Azimuth & Model & $\Psi_{1}[\mathrm{rad}]$ & $\Psi_{2}[\mathrm{rad}]$ & $\Psi_{3}[\mathrm{rad}]$ \\
\hline $0^{\circ}$ & 50 elements, 3 nodes & 0 & -2.7614 & 0 \\
$90^{\circ}$ & 50 elements, 3 nodes & $5.577 \times 10^{-8}$ & -2.7614 & $-4.085 \times 10^{-7}$ \\
$180^{\circ}$ & 50 elements, 3 nodes & $1.408 \times 10^{-8}$ & -2.7614 & $-6.938 \times 10^{-7}$ \\
\hline $0^{\circ}$ & 100 elements, 2 nodes & 0 & -2.7613 & 0 \\
$90^{\circ}$ & 100 elements, 2 nodes & $9.625 \times 10^{-5}$ & -2.7613 & $-1.024 \times 10^{-3}$ \\
$180^{\circ}$ & 100 elements, 2 nodes & $-3.812 \times 10^{-5}$ & -2.7612 & $-1.336 \times 10^{-3}$ \\
\hline $0^{\circ}$ & 10 elements, 3 nodes & 0 & -2.7553 & 0 \\
$90^{\circ}$ & 10 elements, 3 nodes & $2.339 \times 10^{-5}$ & -2.7553 & $-1.621 \times 10^{-4}$ \\
$180^{\circ}$ & 10 elements, 3 nodes & $4.858 \times 10^{-6}$ & -2.7553 & $-3.010 \times 10^{-4}$ \\
\hline $0^{\circ}$ & 20 elements, 2 nodes & 0 & -2.7597 & 0 \\
$15^{\circ}$ & 20 elements, 2 nodes & $5.306 \times 10^{-4}$ & -2.7596 & $-4.583 \times 10^{-3}$ \\
$90^{\circ}$ & 20 elements, 2 nodes & $2.333 \times 10^{-3}$ & -2.7589 & $-2.453 \times 10^{-2}$ \\
$180^{\circ}$ & 20 elements, 2 nodes & $-7.965 \times 10^{-4}$ & -2.7562 & $-3.235 \times 10^{-2}$ \\
\hline
\end{tabular}

Table 2. Tip rotations (in the plane of the undeformed beam) under a tip follower force of $3000 \mathrm{kN}$ for varying azimuth orientations of the undeformed beam in the $x-y$ plane.

The two-dimensional problem is approximated by modeling a rectangular wing of very large aspect-ratio, $\mathcal{O}\left(10^{3}\right)$. For both of the force calculation methods employed, agreement with the linear theories of Theodorsen ${ }^{39}$ and Garrick ${ }^{40}$ is excellent. Previous work by the authors indicates that drag predictions from both force calculation methods convergence similarly with respect to chordwise paneling in two-dimensional problems. ${ }^{29}$

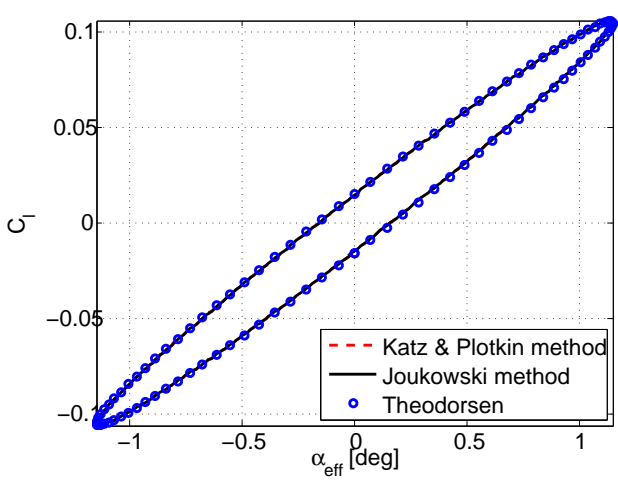

(a) Unsteady lift

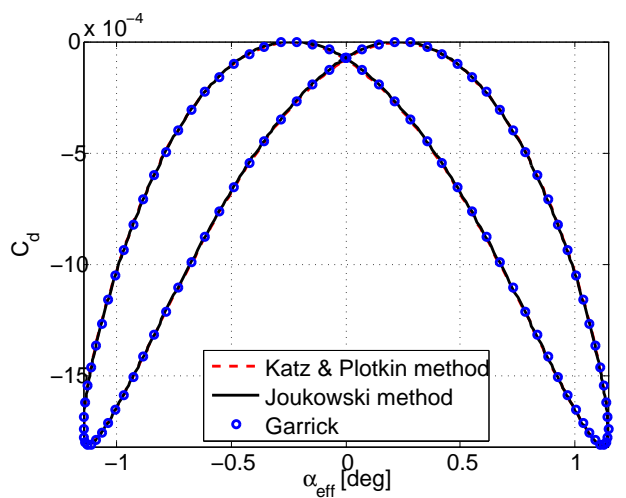

(b) Unsteady drag

Figure 5. Unsteady lift and drag of an airfoil oscillating in plunge with amplitude $\frac{h}{b}=0.2$ and reduced frequency $k=0.1 . M=16$ and $\frac{\Delta t U_{\infty}}{c}=\frac{1}{16}$ in all cases.

In three-dimensional problems the force calculation methods produce very different results when relatively coarse paneling is used. The induced-drag of a pitching (about the quarter-chord) finite-aspect-ratio $(A R=4)$ wing is calculated using the UVLM for a range 
of reduced frequencies including the steady state $(k=0)$. Results are obtained over range of chordwise panelings, $M$, with the number of spanwise panels, $N$, held constant. The results of this study are shown in Fig. 6, which shows that the mean unsteady drag calculated by the Katz \& Plotkin method converges very slowly; using less than 20 chordwise panels will result in errors in the order of $10 \%$. The Joukowski method converges much faster, especially at low reduced frequencies. The error in Fig. 6 has been normalized with respect to the Joukowski method result from the most densely paneled simulation at each reduced frequency.

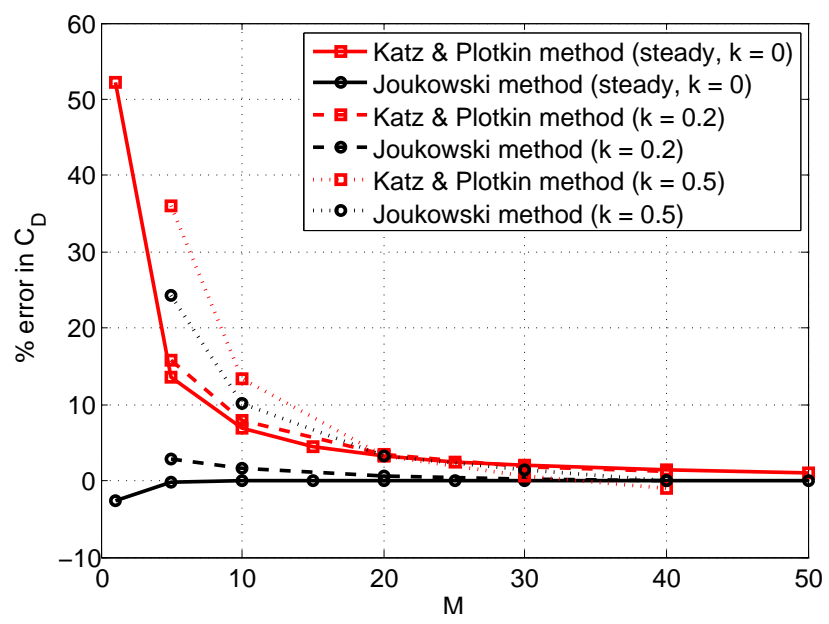

Figure 6. Convergence of steady drag, and mean unsteady drag, with respect to chordwise paneling for a flat-plate of aspect-ratio 4 . Results for $\alpha=5^{\circ} \cos (k s), \frac{\Delta t U_{\infty}}{c}=\frac{1}{M}$, and $N=50$.

The modeling of control surfaces is investigated next by considering an airfoil with a trailing-edge flap that occupies $20 \%$ of the chord. The unsteady lift produced by flap oscillations at $k=0.1$ is predicted using both force calculation methods and compared to the result of Theodorsen. ${ }^{39}$ Here the error was normalized using the modulus of the minimum lift occurring in the cycle. The root-mean-square (RMS) error in lift is shown in Fig. 7(a), which shows both force calculation methods match the linear theory very well, even when only five chordwise panels are used. The error in drag is calculated with respect to the results of Garrick, ${ }^{40}$ and is normalized against the modulus of the minimum drag occurring in the cycle. The resulting RMS error is shown plotted against a range of discretizations in Fig. 7(b). For the case shown both force calculation methods converge slowly; the Katz \& Plotkin method is slightly faster, although the error for a given discretization is of the same order of magnitude regardless of what force calculation method is used. In summary, two-dimensional flapped airfoils require a great many panels to achieve a converged drag result, but as little as five for a converged lift result.

As mentioned above, nonlinear aeroelastic analysis of very flexible wings requires the 


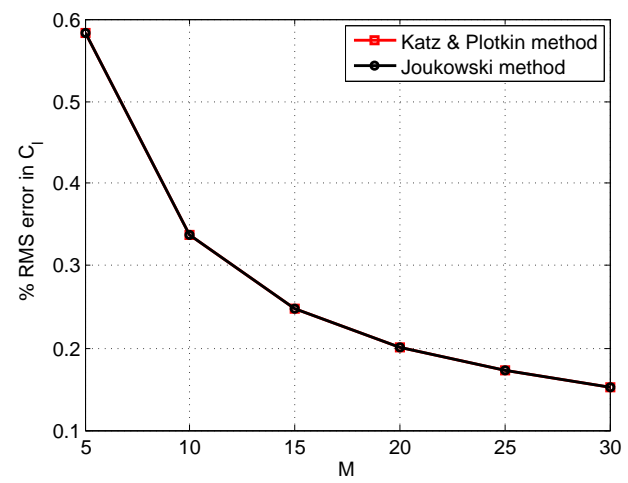

(a) Error in unsteady lift

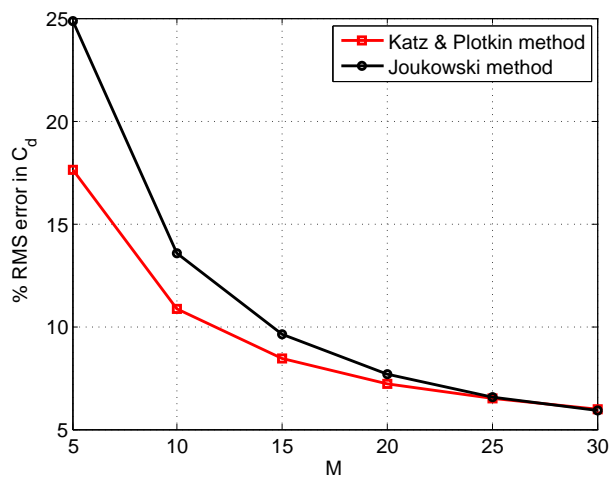

(b) Error in unsteady drag

Figure 7. RMS error in unsteady lift and drag of a flapped airfoil (20\% of chord) oscillating with flap angle amplitude $\beta=0.1^{\circ}$ and reduced frequency $k=0.1$. Error in lift calculated with respect to Theodorsen. Error in drag calculated with respect to Garrick.

instantaneous values of all components of the aerodynamic forces. The results in this section have shown that capturing induced drag requires much finer discretizations than that needed to obtain good estimates of lift. Therefore, discretizations of the lifting surfaces established on the basis of linear analysis may give poor approximations in nonlinear aeroelastic simulations.

\section{Nonlinear Aeroelasticity}

Firstly, a HALE wing, introduced by Patil et al. ${ }^{19}$ and later analyzed by Smith et al. ${ }^{41}$ using a nonlinear-beam / Euler-equation-CFD combination, is analyzed using the present method. The pertinent wing properties are given in table 3 .

\begin{tabular}{ll}
\hline Chord & $1 \mathrm{~m}$ \\
Semi-span & $16 \mathrm{~m}$ \\
Elastic axis & $50 \%$ chord \\
Center of gravity & $50 \%$ chord \\
Mass per unit length & $0.75 \mathrm{~kg} / \mathrm{m}$ \\
Moment of inertia & $0.1 \mathrm{~kg} \cdot \mathrm{m}$ \\
Torsional stiffness & $1 \times 10^{4} \mathrm{~N} \cdot \mathrm{m}^{2}$ \\
Bending stiffness & $2 \times 10^{4} \mathrm{~N} \cdot \mathrm{m}^{2}$ \\
Chordwise bending stiffness & $5 \times 10^{6} \mathrm{~N} \cdot \mathrm{m}^{2}$ \\
\hline
\end{tabular}

Table 3. HALE wing properties.

The static deflection of the wing is analyzed at two angles of attack in a free-stream velocity of $U_{\infty}=25 \mathrm{~m} / \mathrm{s}$. The free-stream density was $\rho_{\infty}=0.08891 \mathrm{~kg} / \mathrm{m}^{3}$ corresponding to the U.S Standard Atmosphere at an altitude of $20 \mathrm{~km}$. 25 load steps are used to linearly approach full aerodynamic load conditions, and the minimum required static residual is set 
to $1 \times 10^{-4}$. 203 -noded beam elements are required for a converged static tip-deflection, corresponding to 40 spanwise panels in the aerodynamic model. 10 chordwise panels are also required, and the steady wake is approximated using vortex rings that stretched 100 chord-lengths downstream. The Joukowski force calculation method, Eqns. (5) \& (6), is used for the aerodynamic analysis.

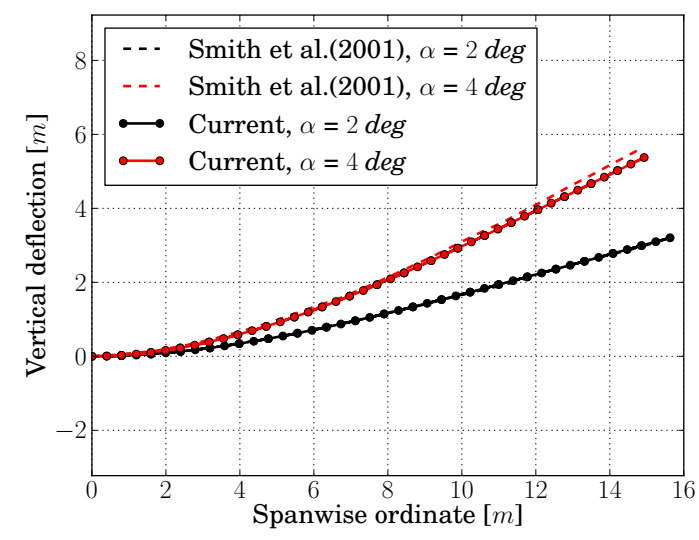

(a) $g=0$

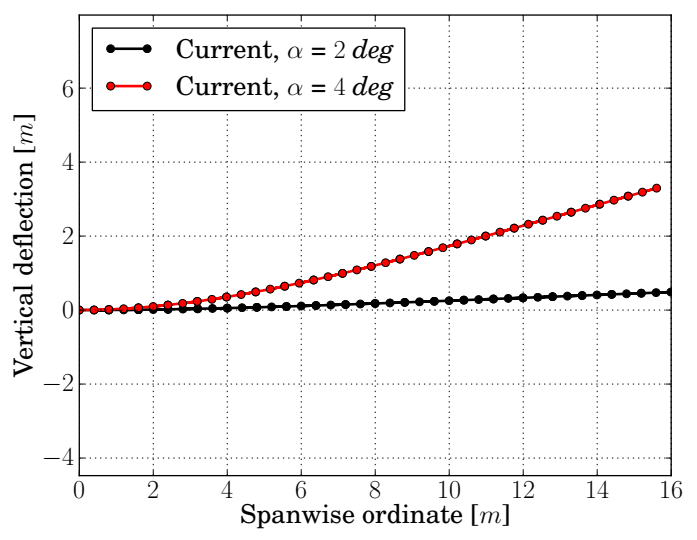

(b) $g=9.754 \mathrm{~m} / \mathrm{s}^{2}$

Figure 8. Vertical deflections of the HALE wing at two angles of attack, with and without the force of gravity. $M=10, N=41$ (20, 3-noded elements).

The results of figure 8(a) show excellent agreement with the work of Smith et al. (2001). This is perhaps to be expected as the free-stream velocity is so low that compressibility effects, present in the Euler equation CFD but not in the vortex-lattice method, are negligible. Perhaps the most significant modeling difference is in the treatment of the wing geometry (NACA0012 section in CFD versus flat-plate in UVLM) especially near the tip - this may explain the discrepancy observed at $\alpha=4 \mathrm{deg}$ in figure 8(a). Since the effect of gravitational forces can be very significant in very flexible wings, they have been included in figure 8(b) for comparison. The acceleration due to gravity used in those results corresponds to that of the U.S Standard Atmosphere at $20 \mathrm{~km}$.

Dynamic aeroelastic analysis is now performed on the Goland wing. ${ }^{18}$ The properties of the wing are defined in table 4. The wing is isotropic, however pitch-plunge coupling occurs due to off-diagonal terms in the sectional mass matrix associated with the aft-displacement of the sectional center of gravity from the elastic axis.

The flutter speed of the Goland wing is calculated by incrementing the free-stream velocity of successive time-marching simulations until the wing-tip response shows neutral stability (undamped oscillations). A small perturbation to the system is introduced by using initial conditions corresponding to the static solution at a very small angle of attack, $\alpha_{t \leq 0}=0.05 \mathrm{deg}$. For $t>0$ the angle of attack is set to zero, introducing a step change sim- 


\begin{tabular}{ll}
\hline Chord & $1.8288 \mathrm{~m}$ \\
Semi-span & $6.096 \mathrm{~m}$ \\
Elastic axis & $33 \%$ chord \\
Center of gravity & $43 \%$ chord \\
Mass per unit length & $35.71 \mathrm{~kg} / \mathrm{m}$ \\
Moment of inertia & $8.64 \mathrm{~kg} \cdot \mathrm{m}$ \\
Torsional stiffness & $0.99 \times 10^{6} \mathrm{~N} \cdot \mathrm{m}^{2}$ \\
Bending stiffness & $9.77 \times 10^{6} \mathrm{~N} \cdot \mathrm{m}^{2}$ \\
\hline
\end{tabular}

Table 4. Goland wing properties.

ilar to that of a small sharp-edged gust. Gravity forces are neglected, and the free-stream density is set as $\rho_{\infty}=1.02 \mathrm{~kg} / \mathrm{m}^{3}$. The numerical damping used in the Newmark method is set to $1 \times 10^{-4}$, and the maximum residual is set to $\epsilon_{1}=1 \times 10^{-5}$. The Katz and Plotkin force calculation is used (the Joukowski method produces very similar results), and the wake length is set as 15 chord-lengths. Loosely-coupled time-integration is used.

\begin{tabular}{llccc} 
Source & Aero model & Beam elements & Chordwise panels & $U_{f}[\mathrm{~m} / \mathrm{s}]$ \\
\hline \hline Current & UVLM & 6,2 -noded & 6 & 164.5 \\
Current & UVLM & 6,2 -noded & 12 & 164.5 \\
Current & UVLM & 6,2 -noded & 18 & 164.5 \\
Current & UVLM & 12, 2-noded & 6 & 168.0 \\
Current & UVLM & 18,2 -noded & 6 & 169.0 \\
\hline Goland $^{42}$ & analytical & N/A & N/A & 137.2 \\
Patil et al. ${ }^{43}$ & strip theory & - & N/A & 135.7 \\
Wang et al. ${ }^{44}$ & UVLM & - & 8 & 163.8 \\
\hline
\end{tabular}

Table 5. Convergence of Goland wing flutter speed with finite element discretizations, and comparison with previously published results.

The flutter speed, $U_{f}$, is calculated to $\pm 0.5 \mathrm{~m} / \mathrm{s}$ using a range of discretizations and the results compiled into table 5 . The number of beam nodes, and hence the number of spanwise panels, is varied, as are the number of chordwise panels. The time step is set according to the relation $\frac{\Delta t U_{\infty}}{c}=\frac{1}{M}$. The coarsest chordwise discretization, $M=6$, is chosen so that the wing response is adequately resolved (in time) at the expected reduced frequency of flutter, $k_{f} \approx 0.37$. In fact, from table 5 , it can be concluded that this resolution is adequate for calculation of the flutter velocity, as increasing the number of chordwise panels makes negligible difference. However, the spanwise discretization (of both the beam and UVLM models) makes a difference; a $3 \%$ change from coarse to fine discretizations is observed, with a converged flutter velocity of $169.0 \mathrm{~m} / \mathrm{s}$. The effect of wake roll-up on the flutter speed was tested, but was found to make negligible difference, despite changes in the wake shape.

The flutter speed calculated by other authors is also shown in table 5. For a wing of such low aspect ratio, the assumption of locally-two-dimensional aerodynamics ${ }^{42,43}$ results in a 
very conservative estimate for flutter velocity. The current methodology, and that of Wang et al. ${ }^{44}$ includes finite-wing effects, hence the good agreement.

Finally, the time-response of the Goland wing is investigated at a velocity well-above flutter speed. Under such a condition, and in the absence of nonlinear restoring forces (e.g, cubic hardening in the structural model, or aerodynamic stall), the wing response should show exponential growth. Contrary to this, when using the Katz and Plotkin method, an apparent limit-cycle oscillation (LCO) is observed, shown in figure 9. This effect is not observed when using the Joukowski method however, also shown in figure 9. Since the beam model is nonlinear, the coupled effect of bending and torsion creates a sideslip relative to the orientation of panels in the aerodynamic model; it is postulated that the difference observed in the wing response is due to the inadequate treatment of this sideslip in the Katz and Plotkin method (Eqns. (7)-(8)). Therefore, for any applications featuring large deformations, such as FAFD analysis, the Joukoswki method is preferred.

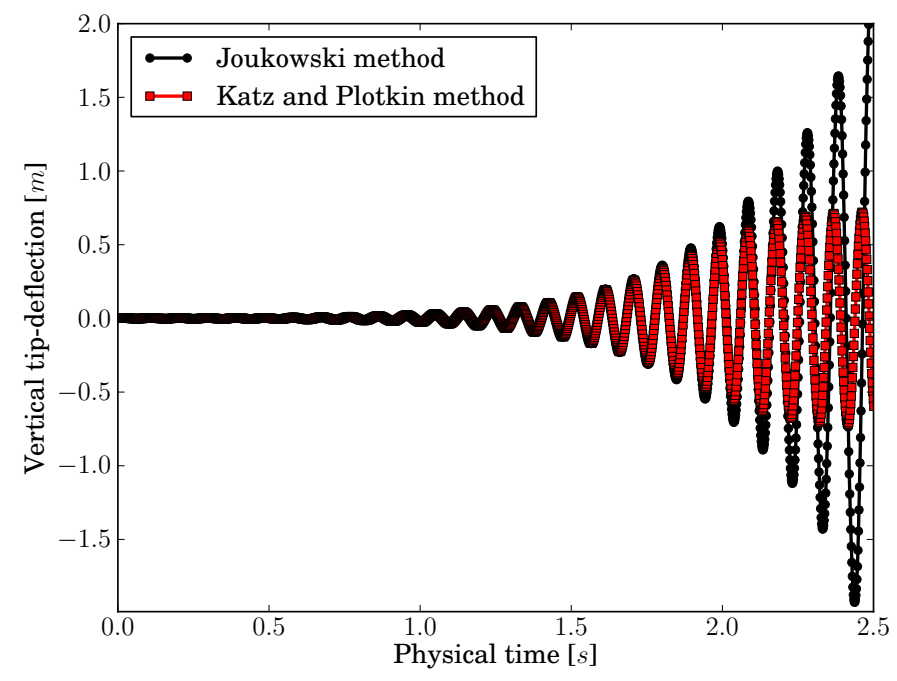

Figure 9. Goland wing tip-response at $U_{\infty}=180 \mathrm{~m} / \mathrm{s}$. Both force calculation methods are shown. $N=24, M=6$ and $\frac{\Delta t U_{\infty}}{c}=\frac{1}{M}$.

\section{Concluding Remarks}

The large deformations and complex kinematics present in the dynamics of very flexible aircraft called for a review of some key analysis methodologies used in flexible aircraft flight dynamics modeling. In particular, the objectivity of the geometrically-exact composite beam (GECB) model, and the force calculations employed in the unsteady vortex lattice method (UVLM), have been shown to be important details in the analysis of very flexible aircraft dynamics. 
The objectivity of the GECB model is tested on a model problem featuring large outof-plane displacements and rotations around all three axes in static equilibrium conditions. Typical errors due to the non-objectivity of the formulation have been exemplified by simply changing the reference frame in which the problem is solved. It is demonstrated that an informed choice of the finite element discretization can help minimize these errors. In particular, 3-noded elements show quadratic convergence with the discretization, thus greatly outperforming 2-noded elements for a given number of structural degrees of freedom in the problem.

Two force calculation methods are presented for the unsteady vortex lattice method (UVLM). The methods are referred to as the Katz and Plotkin method and the Joukowski method, after those who provided the basis on which they are formulated. In two dimensions the unsteady forces predicted using these methods converge similarly with respect to the chordwise discretization used, including in cases featuring dynamic flap deflections. The converged results match those of Theodorsen and Garrick. For the low-aspect-ratio wing studied the Joukoswki method requires far fewer panels for a converged drag result.

Static aeroelastic analysis of a representative HALE-type wing shows the effect of gravity is found to have a dramatic effect on the predicted static deformation, and therefore must be included in future analysis. In addition to this, coupled nonlinear time-marching analysis is applied to the Goland wing and predictions of the flutter speed are found to compare well to published results. The closest agreement is with aeroelastic formulations that also use a three-dimensional aerodynamic model. When using the Katz and Plotkin force calculation method an apparent LCO is observed at a free-stream velocity above the flutter velocity. However, analysis using the Joukoski method shows the exponential growth expected in such conditions. It is therefore postulated that the LCO is observed due to inadequate treatment of sideslip in the Katz and Plotkin method presented in this paper. Hence, the Joukowski method is preferred for applications featuring large deformations, such as flexible aircraft flight dynamics.

\section{Acknowledgments}

This work was supported by the UK Engineering and Physical Sciences Research Council under grant number EP/I014683/1.

\section{References}

${ }^{1}$ Noll, T. E., Brown, J. M., Perez-Davis, M. E., Ishmael, S. D., Tiffany, G. C., and Gaier, M., "Investigation of the Helios Prototype Aircraft Mishap," Mishap Report: Volume 1, NASA, Jan. 2004.

${ }^{2}$ Shearer, C. M. and Cesnik, C. E., "Nonlinear flight dynamics of very flexible aircraft," Journal of 
Aircraft, Vol. 44, No. 5, 2007, pp. 1528 - 1545.

${ }^{3}$ Jian, Z. and Jinwu, X., "Nonlinear Aeroelastic Response of High-aspect-ratio Flexible Wings," Chinese Journal of Aeronautics, Vol. 22, No. 4, Aug. 2009, pp. 355 - 363.

${ }^{4}$ Wang, Z., Chen, P. C., Liu, D. D., and Mook, D. T., "Nonlinear-Aerodynamics/Nonlinear-Structure Interaction Methodology for a High-Altitude Long-Endurance Wing," Journal of aircraft, Vol. 47, No. 2, 2010 , pp. $556-566$.

${ }^{5} \mathrm{Su}$, W. and Cesnik, C. E. S., "Dynamic Response of Highly Flexible Flying Wings," AIAA Journal, Vol. 49, No. 2, 2011, pp. 324 - 339.

${ }^{6}$ Dillsaver, M. J., Cesnik, C. E. S., and Kolmanovsky, I. V., "Gust Response Sensitivity Characteristics of Very Flexible Aircraft," AIAA Atmospheric Flight Mechanics Conference, AIAA, Minneapolis, Minnesota, Aug. 2012.

${ }^{7}$ Patil, M. J., Hodges, D. H., and Cesnik, C. E., "Nonlinear aeroelasticity and flight dynamics of highaltitude long-endurance aircraft," Journal of Aircraft, Vol. 38, No. 1, 2001, pp. 88 - 94.

${ }^{8}$ Palacios, R., Murua, J., and Cook, R., "Structural and aerodynamic models in nonlinear flight dynamics of very flexible aircraft," AIAA Journal, Vol. 48, No. 11, 2010, pp. 2648 - 2659.

${ }^{9}$ Crisfield, M. A. and Jelenić, G., "Objectivity of strain measures in the geometrically exact threedimensional beam theory and its finite-element implementation," Proceedings of the Royal Society of London. Series A: Mathematical, Physical and Engineering Sciences, Vol. 455, No. 1983, 1999, pp. 1125 - 1147.

${ }^{10}$ Leishman, J. G., "Unsteady lift of a flapped airfoil by indicial concepts," Journal of Aircraft, Vol. 31, No. 2, 1994, pp. $288-297$.

${ }^{11}$ Peters, D., "Two-dimensional incompressible unsteady airfoil theory - An overview," Journal of Fluids and Structures, Vol. 24, No. 3, 2008, pp. $295-312$.

${ }^{12}$ Katz, J. and Plotkin, A., Low-Speed Aerodynamics, Cambridge University Press, 2001.

${ }^{13}$ Albano, E. and Rodden, W., "A doublet-lattice method for calculating lift distributions on oscillating surfaces in subsonic flows." AIAA Journal, Vol. 7, No. 2, 1969, pp. $279-285$.

${ }^{14}$ Elzebda, J. M., Mook, D. T., and Nayfeh, A. H., "Numerical simulation of steady and unsteady, vorticity-dominated aerodynamic interference," Journal of Aircraft, Vol. 31, No. 5, 1994, pp. 1031-1036.

${ }^{15}$ Murua, J., Palacios, R., and Graham, J. M. R., "Assessment of Wake-Tail Interference Effects on the Dynamics of Flexible Aircraft," AIAA Journal, Vol. 50, 2012, pp. 1575 - 1585.

${ }^{16}$ Murua, J., Palacios, R., and Graham, J. M. R., "Applications of the unsteady vortex-lattice method in aircraft aeroelasticity and flight dynamics," Progress in Aerospace Sciences, Vol. 55, Nov. 2012, pp. 46 72 .

${ }^{17}$ Hesse, H. and Palacios, R., "Consistent structural linearisation in flexible-body dynamics with large rigid-body motion," Computers $\& 3$ Structures, Vol. 110 - 111, 2012, pp. 1-14.

${ }^{18}$ Goland, M., "The Flutter of a Uniform Cantilever Wing," Journal of Applied Mechanics, Vol. 12, No. 4, Dec. 1945, pp. A197 - A208.

${ }^{19}$ Patil, M. J. and Hodges, D. H., "On the importance of aerodynamic and structural geometrical nonlinearities in aeroelastic behavior of high-aspect-ratio wings," Journal of Fluids and Structures, Vol. 19, No. 7, 2004, pp. $905-915$.

${ }^{20}$ Hodges, D. H., "A mixed variational formulation based on exact intrinsic equations for dynamics of moving beams," International Journal of Solids and Structures, Vol. 26, No. 11, 1990, pp. 1253 - 1273. 
${ }^{21}$ Géradin, M. and Cardona, A., Flexible Multibody Dynamics: A Finite Element Approach, John Wiley \& Sons Ltd, Chichester, UK, 2001.

${ }^{22}$ Bauchau, O. A., Epple, A., and Heo, S., "Interpolation of finite rotations in flexible multi-body dynamics simulations," Proceedings of the Institution of Mechanical Engineers, Part K: Journal of Multibody Dynamics, Vol. 222, No. 4, 2008, pp. 353 - 366.

${ }^{23}$ Murua, J., Flexible Aircraft Dynamics with a Geometrically-Nonlinear Description of the Unsteady Aerodynamics, Ph.D. thesis, Imperial College London, Department of Aeronautics, May 2012.

${ }^{24}$ Morino, L. and Bernardini, G., "Singularities in BIEs for the Laplace equation; Joukowski trailing-edge conjecture revisited," Engineering Analysis with Boundary Elements, Vol. 25, No. 9, Oct. 2001, pp. 805 818.

${ }^{25}$ Cottet, G. and Koumoutsakos, P., Vortex methods: theory and practice, Cambridge Univ Pr, 2000.

${ }^{26}$ Stanford, B. K. and Beran, P. S., "Analytical Sensitivity Analysis of an Unsteady Vortex-Lattice Method for Flapping-Wing Optimization," Journal of Aircraft, Vol. 47, No. 2, 2010, pp. 647 - 662.

${ }^{27}$ Gogulapati, A., Friedmann, P. P., Kheng, E., and Shyy, W., "Approximate Aeroelastic Modeling of Flapping Wings in Hover," AIAA Journal, Vol. 51, No. 3, March 2013, pp. 1 - 17.

${ }^{28}$ Pesmajoglou, S. D. and Graham, J. M. R., "Prediction of aerodynamic forces on horizontal axis wind turbines in free yaw and turbulence," Journal of Wind Engineering and Industrial Aerodynamics, Vol. 86, No. 1, 2000, pp. 1 - 14 .

${ }^{29}$ Simpson, R. J. S., Palacios, R., and Murua, J., "Induced Drag Calculations in the Unsteady VortexLattice Method," AIAA Journal, 2012, Accepted for publication.

${ }^{30}$ Piperno, S. and Farhat, C., "Partitioned procedures for the transient solution of coupled aeroelastic problems - Part II: energy transfer analysis and three-dimensional applications," Computer methods in applied mechanics and engineering, Vol. 190, No. 24, 2001, pp. 3147 - 3170.

${ }^{31}$ Stanford, B. and Beran, P., "Formulation of Analytical Design Derivatives for Nonlinear Unsteady Aeroelasticity," AIAA Journal, Vol. 49, No. 3, March 2011, pp. 598 - 610.

${ }^{32}$ Perez, R. E., Jansen, P. W., and Martins, J. R. R. A., "pyOpt: a Python-based object-oriented framework for nonlinear constrained optimization," Structural and Multidisciplinary Optimization, Vol. 45, No. 1, 2012, pp. $101-118$.

${ }^{33}$ Martins, J. R. R. A., Marriage, C., and Tedford, N., "pyMDO: An Object-Oriented Framework for Multidisciplinary Design Optimization," ACM Trans. Math. Softw., Vol. 36, No. 4, Aug. 2009, pp. 20:1 $20: 25$.

${ }^{34}$ Gray, J. S., Moore, K. T., Hearn, T. A., and Naylor, B. A., "A Standard Platform for Testing and Comparison of MDAO Architectures," 53rd AIAA/ASME/ASCE/AHS/ASC Structures, Structural Dynamics, and Materials Conference, AIAA, Honolulu, HI, April 2012.

${ }^{35}$ Boschitsch, A. H., Usab Jr, W. J., and Epstein, R. J., "Fast lifting panel method," 14th AIAA Computational Fluid Dynamics Conference, Norfolk, VA, 1999, pp. 1049 - 1075.

${ }^{36}$ Greengard, L. and Rokhlin, V., "A fast algorithm for particle simulations," Journal of Computational Physics, Vol. 73, No. 2, 1987, pp. 325 - 348.

${ }^{37}$ Willis, D. J., Peraire, J., and White, J. K., "A combined pFFT-multipole tree code, unsteady panel method with vortex particle wakes," International Journal for Numerical Methods in Fluids, Vol. 53, No. 8, 2007, pp. $1399-1422$. 
${ }^{38}$ Khosravi, P., Ganesan, R., and Sedaghati, R., "Corotational non-linear analysis of thin plates and shells using a new shell element," International Journal for Numerical Methods in Engineering, Vol. 69, No. 4, 2007, pp. $859-885$.

${ }^{39}$ Theodorsen, T., "General theory of aerodynamic instability and the mechanism of flutter," NACA Report 496, National Advisory Committee for Aeronautics, 1935.

${ }^{40}$ Garrick, I. E., "Propulsion of a flapping and oscillating airfoil," NACA Report 567, National Advisory Committee for Aeronautics, 1937.

${ }^{41}$ Smith, M. J., Patil, M. J., and Hodges, D. H., "CFD-based analysis of nonlinear aeroelastic behavior of high-aspect ratio wings," 42nd AIAA/ASME/ASCE/AHS/ASC Structures, Structural Dynamics, and Materials Conference and Exhibit, Seattle, WA, April 2001.

${ }^{42}$ Goland, M. and Luke, Y. L., "The flutter of a uniform wing with tip weights," Journal of Applied Mechanics, Vol. 15, No. 1, March 1948, pp. $13-20$.

${ }^{43}$ Patil, M. J., Hodges, D. H., and Cesnik, C. E., "Nonlinear aeroelastic analysis of complete aircraft in subsonic flow," Journal of Aircraft, Vol. 37, No. 5, 2000, pp. $753-760$.

${ }^{44}$ Wang, Z., Chen, P. C., Liu, D. D., Mook, D. T., and Patil, M. J., "Time domain nonlinear aeroelastic analysis for hale wings," 4 7th AIAA/ASME/ASCE/AHS/ASC Structures, Stuctural Dynamics and Materials Conference, Newport, Rhode Island, May 2006. 